\title{
Gain-of-function SNPs in NLRP3 and $I L 1 B$ genes confer protection against obesity and T2D: undiscovered role of inflammasome genetics in metabolic homeostasis?
}

\author{
Juliana de Moraes Rodrigues ${ }^{1} \cdot$ Dhemerson Souza de Lima $^{1} \cdot$ Vinicius N. C. Leal $^{1}$ • \\ Adriana A. Bosco ${ }^{2}$ Valeria Sandrim ${ }^{3}$ - Alessandra Pontillo ${ }^{1}$
}

Received: 31 March 2017 / Accepted: 1 June 2017 / Published online: 20 June 2017

(C) Springer Science+Business Media, LLC 2017

\section{Introduction}

Inflammation represents a cause, but also a consequence of obesity, contributing to disrupt metabolic homeostasis. Previously associated to high fat diets and physical inactivity, the contribution of gut microbiota and genetic inheritance are also being evaluated as risk factors for obesity. [1]

Increasing, and sometimes contrasting, findings have revealed the involvement of inflammasome in the development of obesity and obesity-associated diseases in experimental models. Inflammasome is a cytoplasmic complex responsible for the activation of caspase- 1 and the consequent production of the biologic active form of interleukin (IL)-1ß and IL-18. Several intracellular innate receptors (i.e., NLR Family Pyrin Domain Containing 1/ NLRP1, NLRP3, NLRP6, Absent in Melanoma 2/AIM2) sense pathogen-associated and danger-associated molecular patterns (DAMPs), respectively, and became able to mount an inflammasome. These molecules are expressed in

Electronic supplementary material The online version of this article (doi:10.1007/s12020-017-1343-0) contains supplementary material, which is available to authorized users.

$\triangle$ Alessandra Pontillo

pontillo.a@gmail.com

1 Laboratório de Imunogenética, Departamento of Imunologia, Instituto de Ciências Biomédicas, Universidade de São Paulo (USP), São Paulo, SP, Brazil

2 Nucleo de Pos-Graduacao e Pesquisa-Santa Casa de Belo Horizonte, Belo Horizonte, MG, Brazil

3 Departmento de Farmacologia, Instituto de Biociencias, Universidade Estadual Paulista/UNESP, Botucatu, SP, Brazil leucocytes, as well as in non-immune cells, such as adipose tissue and epithelium. In intestinal epithelial cells (IEC), it has been demonstrated that specific innate receptors act not only fighting against pathogens, but also regulating gut microbiota [1].

Moreover, once obesity has been established, several obesity-related DAMPs (i.e., high levels of glucose, fatty acids, uric acid), oxidative and/or endoplasmic reticulum (ER) stress are responsible for inflammasome-dependent secretion of IL-1B and IL-18 from both leucocytes and adipocytes, leading to a dangerous pro-inflammatory loop [2].

In humans, it is possible to observe an increasing of both inflammasome cytokines in serum from obese individuals $[3,4]$. However, it is difficult to understand whether the activation of inflammasome has been the trigger or, eventually, an important contributing factor for the development of obesity, or whether it "simply" amplifies the metabolic stress induced inflammation.

The aim of this study was to elucidate the contribution of inflammasome genetics in the development of obesity and obesity-associated morbidities.

\section{Material and methods}

\section{Patients}

Two hundred and eighty eight unrelated obese patients (male/female: 22/266) and 96 non-obese individuals (male/ female: 19/77) were recruited from the Department of Obesity, Centre of Medical Specialties of Minas Gerais (Belo Horizonte, Southeast of Brazil) in compliance with the Institutional Review Board of the "Santa Casa de Belo 
Horizonte" hospital. Body mass index (BMI) was used to define obesity: subjects with $\mathrm{BMI} \geq 30 \mathrm{~kg} / \mathrm{m}^{2}$ were classified as obese, BMI of $25-29.9 \mathrm{~kg} / \mathrm{m}^{2}$ as over-weight and BMI of $18.5-24.9 \mathrm{~kg} / \mathrm{m}^{2}$ as normal weight [5]. Due to the limited number of over-weight individuals in our cohort $(n=3)$, and considering their border-line BMI values (25.03; 25.33 and 27.10, respectively), they were grouped with the normal-weight group. Median BMI of obese group is $45.17 \mathrm{Kg} / \mathrm{m}^{2}$ (min: 30.41-max: 82.83 ), and of non-obese group is $22.68 \mathrm{Kg} / \mathrm{m}^{2}$ (min: 17.90-max: 27.10 ).

To determine metabolic risk factors in obese subjects, we followed the diagnostic criteria for metabolic syndrome defined in accordance with the American Heart Association/ National Heart, Lung, and Blood Institute Scientific Statement [6], which included systemic arterial hypertension (SAH) (systolic blood pressure $>140 \mathrm{mmHg}$, and/or diastolic blood pressure $>90 \mathrm{mmHg}$, and/or drug treatment for elevated blood pressure) and type-2 diabetes (T2D) (elevated fasting glucose $\geq 100 \mathrm{mg} / \mathrm{dL}$ or drug treatment for elevated glucose). One hundred and six out of 288 obese, and 35 out of 96 non-obese individuals have SAH. Seventy three (male/female: 6/67) out of 288 obese, and 6 (male/ female: 1/5) out of 96 non-obese individuals have T2D. For 18 obese individuals (male/female: $3 / 15$ ) we did not have clinical data about T2D. Sixty seven (male/female: 6/61) out of 288 obese, and 5 (male/female: 1/4) out of 96 nonobese individuals have both SAH and T2D.

\section{SNPs genotyping}

Five SNPs were selected based on minor allele frequency, functional effect and/or previously reported association with human disorders: NLRP1 rs 12150220 (L155H), rs11651270 (M1184V); NLRP3 rs10754558 (3'UTR); IL1B rs16944 (promoter); IL18 rs1834481 (non-coding transcript exon variant/intron) [7]. SNPs genotyping was performed using allele-specific Taqman assays (Applied Biosystems) and qPCR using the QuantStudio3 Real-Time PCR platform (Applied Biosystems).

\section{Data analyses}

The effect of SNPs on clinical and biochemical variables was analysed by multivariate association based on general linear model adjusted for confounders variables (age, sex, ethnicity) using R-project package "SNP-assoc" version 1.9-2. Analyses were performed in the codominant, dominant, recessive and overdominant models for all SNPs. The Haploview software was used to investigate the linkage disequilibrium (LD) and to derive the haplotypes. A significant threshold of $p=0.01$ has been assumed after Bonferroni correction for multiple SNPs analysis $(p=0.05$ / $\mathrm{n} ; n=5$ SNPs).

\section{Results and discussion}

In the present study, we evaluated the genetic contribution of inflammasome-encoding genes NLRPI, NLRP3, ILIB, ILI8 in the development of obesity and obesity-associated comorbidities, precisely SAH and T2D. All the polymorphisms resulted in Hardy-Weinberg equilibrium. No LD was found for NLRPI SNPs.

Nor NLRPI nor ILI8, which have been recently showed to play a key role in obesity in animal model $[8,9]$, were associated to obesity or related diseases, at least in our cohort (Supplementary File 1).

On the other hand, NLRP3 gain-of-function variant rs 10754558 resulted significantly associated to obesity ( $\mathrm{p}_{\text {adj }}$ $=0.009$ ) (Table 1). NLRP3 rs10754558 G minor allele resulted more frequent in non-obese than in obese individuals $(\mathrm{C} / \mathrm{G}+\mathrm{G} / \mathrm{G}: 0.68$ vs. 0.55 ; $\mathrm{OR}=0.46$, according to a dominant model of inheritance) (Table 1), suggesting a protective role of this variant against obesity development.

When BMI, as a continuous variable, was considered, NLRP3 rs10754558 continued to be associated with obesity. Individuals carrying NLRP3 rs10754558 C/G genotype presented lower BMI values compared to homozygotes $(\mathrm{C} /$ G: $38.87 \pm 1.01$ vs. $\mathrm{C} / \mathrm{C}+\mathrm{G} / \mathrm{G}: 42.40 \pm 0.94 ; \mathrm{p}_{\mathrm{adj}}=0.015$, according to an over-dominant model of inheritance) (Table 1).

The protective role of this NLRP3 rs10754558 variant in obesity is quite unexpected. rs 10754558 SNP is localized in $3^{\prime} \mathrm{UTR}$ region of NLRP3 and determines an increased NLRP3 mRNA stability possibly affecting the micro RNA223 binding site, cooperating to increase transduction rate of NLRP3 mRNA [10]. It increases the secretion of IL-1ß and IL-18 by human peripheral blood monocytes or monocyte-derived macrophages stimulated with bacterial lipopolysaccharides (yet unpublished data).

Activation of NLRP3 inflammasome and the resulting production of pro-inflammatory cytokines have been showed to play a major role in adipogenesis and in proinflammatory transformation of adipose tissue in chronical overfeeding mice [11].

However, we must consider the physiological role of inflammasome [12] in maintaining body homeostasis. A basal activation of inflammasomes is required to control microbiota, IEC proliferation and tissue repair through the production of IL-18 [13]. IL-18 appeared to exert a paradoxical effect in obesity: obese individuals present increased serum level of the cytokine [3, 14], nonetheless it has been recently demonstrated that IL-18 represents a major player against obesity [8, 9]. Vijay-Kumar and Gewirtz discussed the role of NLRP3 in obesity, emphasizing that the deficiency of NLRP3 leads to an altered microbiota predisposing to the development of obesity and obesity-associated morbidities in mice [15]. Altogether 
Table 1 Detailed results for association of NLRP3 rs10754558 and IL1B rs16944 with obesity and type-2 diabetes

\begin{tabular}{|c|c|c|c|c|}
\hline Rs10754558 & Obese $(n=288)$ & Non-obese $(n=96)$ & $\mathrm{p}_{\text {adj }}$ & $\mathrm{OR}_{\text {adj }}(95 \% \mathrm{CI})$ \\
\hline $\mathrm{C} / \mathrm{C}$ & 0.45 & 0.32 & 0.009 (dom) & Ref \\
\hline $\mathrm{C} / \mathrm{G}+\mathrm{G} / \mathrm{G}$ & 0.55 & 0.68 & & $0.46(0.25-0.84)$ \\
\hline Rs10754558 & Individuals (Frequency) & BMI $($ Mean \pm SE $)$ & $\mathrm{p}_{\text {adj }}$ & Dif (low/up) \\
\hline $\mathrm{C} / \mathrm{C}+\mathrm{G} / \mathrm{G}$ & 0.63 & $42.40 \pm 0.94$ & 0.007 (over) & -3.69 \\
\hline $\mathrm{C} / \mathrm{G}$ & 0.37 & $38.87 \pm 1.01$ & & $(-6.35 /-1.03)$ \\
\hline Rs16944 & No $\mathrm{T} 2 \mathrm{D}(n=215)$ & $\mathrm{T} 2 \mathrm{D}(n=73)$ & $\mathrm{p}_{\mathrm{adj}}$ & $\mathrm{OR}_{\mathrm{adj}}(95 \% \mathrm{CI})$ \\
\hline $\mathrm{G} / \mathrm{G}$ & 0.33 & 0.49 & $0.024(\mathrm{dom})$ & Ref \\
\hline $\mathrm{G} / \mathrm{A}+\mathrm{A} / \mathrm{A}$ & 0.67 & 0.51 & & $0.49(0.32-0.92)$ \\
\hline Rs16944 & No T2D female $(n=184)$ & T2D female $(n=67)$ & $\mathrm{p}_{\text {adj }}$ & $\mathrm{OR}_{\mathrm{adj}}(95 \% \mathrm{CI})$ \\
\hline $\mathrm{G} / \mathrm{G}$ & 0.37 & 0.59 & $0.001(\mathrm{dom})$ & Ref \\
\hline $\mathrm{G} / \mathrm{A}+\mathrm{A} / \mathrm{A}$ & 0.63 & 0.41 & & $0.41(0.24-0.71)$ \\
\hline Rs16944 & No T2D $>40$ years $(n=90)$ & T2D $>40$ years $(n=57)$ & $\mathrm{p}_{\mathrm{adj}}$ & $\mathrm{OR}_{\mathrm{adj}}(95 \% \mathrm{CI})$ \\
\hline $\mathrm{G} / \mathrm{G}$ & 0.60 & 0.39 & 0.009 (dom) & Ref \\
\hline $\mathrm{G} / \mathrm{A}+\mathrm{A} / \mathrm{A}$ & 0.40 & 0.61 & & $0.44(0.23-0.83)$ \\
\hline
\end{tabular}

NLRP3 rs10754558 and IL1B rs16944 association with obesity and type 2 diabetes (T2D) respectively, was analysed by general linear model (GLM). Genotypes distribution in obese $(n=288$; BMI $\geq 30)$ and non-obese $(n=96$; BMI $<30)$ individuals, $p$-value adjusted for sex, age, race $\left(\mathrm{p}_{\text {adj }}\right.$ ), Odds Ratio and respective $95 \%$ confidence intervals are reported. The analysis of association of rs16944 with T2D is made only in obese subjects. Model of inheritance (according with Akaike information criterion index) is indicated within brackets (dom dominant, over overdominant)

these findings help us to explain our quite unexpected result.

Finally, when we have considered obesity-related comorbidities, namely $\mathrm{SAH}$ or $\mathrm{T} 2 \mathrm{D}$, we observed no association among SNPs and SAH (Supplementary File 1), but a significant association between $I L 1 B$ rs16944 and T2D (Table 1).

$I L 1 B$ rs16944 minor A allele resulted less frequent in T2D ( $n=73$; male/female: $6 / 67)$ than in non-T2D $(n=215$; male/female: $31 / 184)$ patients (G/A+A/A: 0.51 vs. 0.67; $\left.\mathrm{p}_{\mathrm{adj}}=0.008 ; \mathrm{OR}=0.49\right)$, especially in women $\left(\mathrm{p}_{\mathrm{adj}}=\right.$ 0.001 ; OR $=0.41)$, or in older individuals $(>40$ years; T2D: 57 out of 73; non-T2D: 90 out of 215$)\left(\mathrm{p}_{\mathrm{adj}}=0.009\right.$; $\mathrm{OR}=$ $0.44)$.

Once again, a gain-of-function of inflammasome was associated with protection against the development of diabetes. In mice, IL-1 $\beta$ has been pointed out as one of the factors involved in the translation of obesity-associated inflammation into insulin resistance (as largely reviewed in ref. [16]).

All this considered, we hypothesized two scenarios, one in which the genetic background shapes a predisposing (loss of NLRP3 or NLRP6 in mice [17]) or protective (gain-offunction polymorphisms in inflammasome genes) backbone against obesity; and another, in which, in a gain-of-weight environment, the NLRP3-inflammasome senses cell stress agents and heavily contributes to adipose tissue inflammation [11] and consequently to the pathogenesis of obesity and metabolic disorders.
Funding This study was supported by Sao Paulo Research Foundation (FAPESP) (Grants numbers: 2015/23395-6;) and "Conselho Nacional de Desenvolvimento Cientıfico e Tecnologico" (CNPq). A.P. is a recipient of a CNPq fellowship. J.M.R. was a recipient of a PIBIC Fellowship. V.N.C.L. is a recipient of a FAPESP-funded fellowship. D.L. is a recipient of a UFAM-funded PhD Fellowship.

\section{Compliance with ethical standards}

Conflict of interest The authors declare that they have no competing interests.

Ethical approval Research involved human participants. All procedures performed were in accordance with the ethical standards of the Institutional Review Board of the "Santa Casa de Belo Horizonte" hospital, and with the 1964 Helsinki declaration and its later amendments or comparable ethical standards.

Informed consent Informed consent was obtained from all individual participants included in the study.

\section{References}

1. L.P. Zambetti, A. Mortellaro, NLRPs, microbiota, and gut homeostasis: unravelling the connection. J. Pathol. 233(4), 321-330 (2014)

2. M. Haneklaus, L.A. O'Neill, NLRP3 at the interface of metabolism and inflammation. Immunol. Rev. 265(1), 53-62 (2015)

3. K. Esposito, A. Pontillo, M. Ciotola, C. Di Palo, E. Grella, G. Nicoletti, D. Giugliano, Weight loss reduces interleukin-18 levels in obese women. J. Clin. Endocrinol. Metab. 87(8), 3864-3866 (2002) 
4. A. Satpathy, S. Ravindra, S. Thakur, S. Kulkarni, A. Porwal, S. Panda, Serum interleukin- $1 \beta$ in subjects with abdominal obesity and periodontitis. Obes. Res. Clin. Pract 9(5), 513-521 (2015)

5. K.M. Flegal, B.K. Kit, H. Orpana, B.I. Graubard, Association of all-cause mortality with overweight and obesity using standard body mass index categories: a systematic review and metaanalysis. JAMA 309(1), 71-82 (2013)

6. S.M. Grundy, J.I. Cleeman, S.R. Daniels, K.A. Donato, R.H. Eckel, B.A. Franklin, D.J. Gordon, R.M. Krauss, P.J. Savage, S.C. Smith Jr, J.A. Spertus, F. Costa; American Heart A, National Heart L, Blood I, Diagnosis and management of the metabolic syndrome: an American heart association/national heart, lung, and blood institute scientific statement. Circulation 112(17), 2735-2752 (2005)

7. Y. Zhong, A. Kinio, M. Saleh, Functions of NOD-like receptors in human diseases. Front. Immunol. 4, 333 (2013)

8. A.J. Murphy, M.J. Kraakman, H.L. Kammoun, D. Dragoljevic, M.K. Lee, K.E. Lawlor, J.M. Wentworth, A. Vasanthakumar, M. Gerlic, L.W. Whitehead, L. DiRago, L. Cengia, R.M. Lane, D. Metcalf, J.E. Vince, L.C. Harrison, A. Kallies, B.T. Kile, B. A. Croker, M.A. Febbraio, S.L. Masters, IL-18 production from the NLRP1 inflammasome prevents obesity and metabolic syndrome. Cell. Metab. 23(1), 155-164 (2016)

9. M.G. Netea, L.A. Joosten, The NLRP1-IL18 connection: a stab in the back of obesity-induced inflammation. Cell. Metab. 23(1), 6-7 (2016)

10. Y. Hitomi, M. Ebisawa, M. Tomikawa, T. Imai, T. Komata, T. Hirota, M. Harada, M. Sakashita, Y. Suzuki, N. Shimojo, Y. Kohno, K. Fujita, A. Miyatake, S. Doi, T. Enomoto,
M. Taniguchi, N. Higashi, Y. Nakamura, M. Tamari, Associations of functional NLRP3 polymorphisms with susceptibility to foodinduced anaphylaxis and aspirin-induced asthma. J. Allergy. Clin. Immunol. 124(4), 779-785 (2009). e6

11. R. Stienstra, J.A. van Diepen, C.J. Tack, M.H. Zaki, F.L. van de Veerdonk, D. Perera, G.A. Neale, G.J. Hooiveld, A. Hijmans, I. Vroegrijk, S. van den Berg, J. Romijn, P.C. Rensen, L.A. Joosten, M.G. Netea, T.D. Kanneganti, Inflammasome is a central player in the induction of obesity and insulin resistance. Proc. Natl Acad. Sci. USA 108(37), 15324-15329 (2011)

12. E. Elinav, J. Henao-Mejia, R.A. Flavell, Integrative inflammasome activity in the regulation of intestinal mucosal immune responses. Mucosal Immunol. 6(1), 4-13 (2013)

13. M. Levy, E. Blacher, Elinav E microbiome, metabolites and host immunity. Curr. Opin. Microbiol. 35, 8-15 (2016)

14. J. Hung, B.M. McQuillan, C.M. Chapman, P.L. Thompson, J.P. Beilby, Elevated interleukin-18 levels are associated with the metabolic syndrome independent of obesity and insulin resistance. Arterioscler. Thromb. Vasc. Biol. 25(6), 1268-1273 (2005)

15. M. Vijay-Kumar, A.T. Gewirtz, Is predisposition to NAFLD and obesity communicable? Cell. Metab. 15(4), 419-420 (2012)

16. C. Bing, Is interleukin- $1 \beta$ a culprit in macrophage-adipocyte crosstalk in obesity? Adipocyte 4(2), 149-152 (2015)

17. J.1 Henao-Mejia, E. Elinav, C. Jin, L. Hao, W.Z. Mehal, T. Strowig, C.A. Thaiss, A.L. Kau, S.C. Eisenbarth, M.J. Jurczak, J.P. Camporez, G.I. Shulman, J.I. Gordon, H.M. Hoffman, R.A. Flavell, Inflammasome-mediated dysbiosis regulates progression of NAFLD and obesity. Nature 482(7384), 179-185 (2012) 